\title{
General flux to a trap in one and three dimensions
}

\author{
Robert M Ziff ${ }^{1}$, Satya N Majumdar ${ }^{2}$ and Alain Comtet ${ }^{2,3}$ \\ ${ }^{1}$ Michigan Center for Theoretical Physics and Department of Chemical Engineering, University of \\ Michigan, Ann Arbor, MI 48109-2136, USA \\ ${ }^{2}$ Laboratoire de Physique Théorique et Modèles Statistiques, Université Paris-Sud, Bâtiment 100, \\ 91405 Orsay Cedex, France \\ ${ }^{3}$ Institut Henri Poincaré, 11 rue Pierre et Marie Curie, 75005 Paris, France \\ E-mail: rziff@umich.edu,majumdar@lptms.u-psud.fr and comtet@ipno.in2p3.fr
}

Received 28 June 2006

Published 22 January 2007

Online at stacks.iop.org/JPhysCM/19/065102

\begin{abstract}
The problem of the flux to a spherical trap in one and three dimensions, for diffusing particles undergoing discrete-time jumps with a given radial probability distribution, is solved in general, verifying the Smoluchowski-like solution in which the effective trap radius is reduced by an amount proportional to the jump length. This reduction in the effective trap radius corresponds to the Milne extrapolation length.
\end{abstract}

\section{Introduction}

A classical problem in diffusion and reaction theory is the flux of particles, initially uniform in space, to a trap. When the particles are moving by simple diffusion, this flux can be found by solving the diffusion equation with an adsorbing boundary at the surface of the trap. For example, for a system with a spherical trap of radius $R$, the time-dependent density $\rho(\vec{r}, t)$ evolves via the diffusion equation, $\partial_{t} \rho=D \nabla^{2} \rho$ ( $D$ being the diffusion coefficient), starting from the initial condition $\rho(\vec{r}, 0)=\rho_{0}$ for $r>R$ and $\rho(\vec{r}, 0)=0$ for $r \leqslant R$. The diffusion equation is subject to the boundary conditions $\rho(\vec{r}, t)=0$ for $r=R$ and $\rho(\vec{r}, t) \rightarrow \rho_{0}$ as $r \rightarrow \infty$, for all time $t$. For spatial dimensions $d>2$, the density profile outside the sphere approaches a steady state as $t \rightarrow \infty$, reflecting the fact that Brownian motion is transient for $d>2$ [1]. For example, in three dimensions, the full time-dependent density profile (which is spherically symmetric) can be explicitly obtained for all $t$,

$$
\rho(r, t)=\frac{\rho_{0}}{r}\left[r-R \operatorname{erfc}\left(\frac{r-R}{\sqrt{4 D t}}\right)\right] \quad \text { for } r \geqslant R
$$

where $\operatorname{erfc}(z)=(2 / \sqrt{\pi}) \int_{z}^{\infty} \mathrm{e}^{-u^{2}} \mathrm{~d} u$. In particular, as $t \rightarrow \infty$, the density approaches a stationary profile outside the sphere,

$$
\rho(r, \infty)=\frac{\rho_{0}}{r}(r-R) .
$$


The flux $\Phi(t)=\left.4 \pi R^{2} D(\partial \rho / \partial r)\right|_{r=R}$, defined as the number of particles falling into the trap per unit time, can be easily obtained from $\rho(r, t)$ in equation (1) and one gets the classical result $[2,3]$,

$$
\Phi(t)=4 \pi R D \rho_{0}\left[1+\frac{R}{\sqrt{\pi D t}}\right] .
$$

On the other hand, when the particles are moving by discrete jumps at every time step $\tau$ (as opposed to undergoing continuous-time Brownian motion), the above results for the stationary density profile in equation (2) as well as that for the flux in equation (3) become modified. In [4] this discrete-jump problem was studied by an iterative numerical scheme, for particles undergoing fixed-length jumps of length $\ell$ and random angles (the so-called Rayleigh flights). It was found that in $d=3$, while the density profile $\rho_{n}(r)$ after $n$ time steps approaches a stationary limit as $n \rightarrow \infty$, the stationary profile $\rho_{\infty}(r)$ is different from its continuous-time counterpart in equation (2). Very far from the surface of the sphere, the stationary profile behaves as

$$
\rho_{\infty}(r) \approx \frac{\rho_{0}}{r}[r-(R-\epsilon)] \quad \text { for } r \gg R,
$$

where $\epsilon=c \ell$ and the dimensionless constant $c=0.29795219 \ldots$ was determined numerically [4]. On the other hand, on the surface of the sphere, the stationary density profile approaches a positive value

$$
\rho_{\infty}(R) \approx 0.408245 \frac{\rho_{0} \ell}{R},
$$

where the constant 0.408245 was evaluated numerically in [4]. This is in marked contrast to the continuous-time Brownian case where the stationary density profile vanishes on the surface of the sphere. The distance $\epsilon=c \ell$ is the 'Milne extrapolation length' [5-7] that represents the distance inside the surface where the far steady-state solution in equation (4) extrapolates to zero. The same length $\epsilon$ also appears in the expression for flux [4]

$$
\Phi(t) \approx 4 \pi D \rho_{0}(R-\epsilon)\left[1+\frac{R-\epsilon}{\sqrt{\pi D t}}+\mathcal{O}\left(t^{-3 / 2}\right)\right],
$$

for large $t$. This result was obtained numerically in [4].

Recently we have rigorously proven the result in equation (4) for the large-distance stationary profile and found an analytical expression for $c$ [8]:

$$
c=-\frac{1}{\pi} \int_{0}^{\infty} \frac{\mathrm{d} k}{k^{2}} \ln \left[\frac{6}{k^{2}}\left(1-\frac{\sin k}{k}\right)\right]=0.29795219028 \ldots
$$

Remarkably, the same constant also appeared in the leading finite-size correction to the expected maximum of a discrete-time random walker moving on a continuous line where at each time step the walker jumps by a distance $\xi$ that is chosen from a uniform distribution [9, 10]. In [9], the constant was evaluated numerically by summing a rather complicated double infinite series. Later an exact expression as in equation (7) was derived in [10]. In [8], we showed why the same constant also appeared in the three-dimensional (3D) discrete flux problem. For the 3D flux problem, it was further proven [8] that the density on the surface of the sphere approaches a constant as in equation (5):

$$
\rho_{\infty}(r)=\frac{1}{\sqrt{6}} \frac{\rho_{0} \ell}{R} .
$$

Thus the constant 0.408245 in equation (5), found numerically in [4], was proven to be simply $1 / \sqrt{6}$. Indeed, in [8], an analytical expression for the full stationary density profile for all $r \geqslant R$ (or rather its Laplace transform) was obtained. 
Having obtained the stationary density profile, the next objective is to prove the expression for the time-dependent flux in equation (6) that was found numerically in [4]. If one uses the form in equation (4) of the steady-state solution far from the sphere in the expression for the flux in the continuous-time diffusion problem, one gets [8] $\Phi=4 \pi r^{2} D\left(\mathrm{~d} \rho_{\infty}(r) / \mathrm{d} r\right)=$ $4 \pi D \rho_{0}(R-\epsilon)$, which is precisely the leading time-independent term in the expression for flux in equation (6). Even though this reproduces correctly the leading term in the flux in equation (6), it is not entirely satisfactory since we are using a definition of flux which is valid only for the continuous-time diffusion equation and not for the discrete-time jump process. Moreover, just the knowledge of the stationary density profile is not enough to prove the leading time-dependent behaviour, namely the $1 / \sqrt{t}$ decay to the stationary flux in equation (6). Thus, to rigorously prove the two leading terms in equation (6), we need to obtain the timedependent solution $\rho_{n}(r)$ of the discrete-time process for large $n$ and then use this solution in an appropriate expression for the discrete flux (see below). This is precisely what is achieved in this paper.

The main achievements of this paper are: (i) to prove rigorously the result for the discrete flux in equation (6) for the two leading terms and then (ii) to generalize the results in equations (4)-(6) to the case where the jump length $\left|\vec{r}-\vec{r}^{\prime}\right|$ from $\vec{r}^{\prime}$ to $\vec{r}$ is not of a fixed length (as in Rayleigh flights) but is a random variable drawn from a distribution $W\left(\left|\vec{r}-\vec{r}^{\prime}\right|\right)$ that is bounded above (with an upper cut-off $<2 R$ ), but is otherwise arbitrary. The stationary density profile for the general case was already calculated in detail in [8] and it was shown that equation (4) is valid for the general case where $\epsilon$ has an explicit expression [8]

$$
\epsilon=-\frac{1}{\pi} \int_{0}^{\infty} \frac{\mathrm{d} k}{k^{2}} \ln \left[\frac{1-\hat{f}(k)}{\sigma^{2} k^{2} / 2}\right],
$$

where $\hat{f}(k)=\int_{-\infty}^{\infty} f(x) \mathrm{e}^{\mathrm{i} k x} \mathrm{~d} x$ is the Fourier transform of the function

$$
f(x)=2 \pi \int_{|x|}^{\infty} W(u) u \mathrm{~d} u,
$$

and $\sigma^{2}=\int_{-\infty}^{\infty} f(x) x^{2} \mathrm{~d} x$ is the second moment of $f(x)$ which is assumed to be finite. In terms of $W(u)$, we have $\sigma^{2}=(2 \pi / 3) \int_{-\infty}^{\infty} W(u) u^{4} \mathrm{~d} u$. For the case of the Rayleigh flight, the jump probability $W(u)=\frac{1}{4 \pi \ell^{2}} \delta(u-\ell)$ in three-dimensional space translates to a uniform jump distribution for $f(x)[11]$,

$$
f(x)= \begin{cases}\frac{1}{2 \ell}, & |x|<\ell \\ 0, & \text { otherwise }\end{cases}
$$

Then $\hat{f}(k)=\sin k \ell /(k \ell)$, and equation (9) reduces to equation (7) with $\epsilon=c \ell$.

In addition, for the general jump distribution $W(z)$, another remarkable universal result was proven [8]. It was shown that the stationary density on the surface of the sphere, properly rescaled by $R$ and $\sigma$, is a universal dimensionless constant [8]

$$
\frac{\rho_{\infty}(R)}{\rho_{0}} \frac{R}{\sigma}=\frac{1}{\sqrt{2}},
$$

which generalizes equation (8). In this paper, we will prove that the asymptotic expression for the discrete flux in equation (6) is also valid for a general jump distribution $W(z)$ with the length $\epsilon$ given by the exact formula in equation (9). 
Apart from the three-dimensional results mentioned above, in this paper we also study the one-dimensional discrete flux problem where the origin is a trap and initially random walkers are distributed uniformly with density $\rho_{0}$ on the positive semi-infinite line. At every discrete time step, each walker jumps independently by a distance $x$ that is chosen from a normalized probability density function $f(x)$ with a finite second moment $\sigma^{2}=\int_{-\infty}^{\infty} f(x) x^{2} \mathrm{~d} x$. Due to the recurrent nature of random walk in one dimension, the density approaches zero everywhere on the positive line at late times and the instantaneous flux to the origin also decays to zero with time. Our exact calculation shows that while the instantaneous flux decays algebraically as $1 / \sqrt{n}$ for large time $n$, the cumulative flux $\Phi_{n}^{\text {cum }}$ (i.e., the total number of particles absorbed by the origin up to $n$ steps) behaves asymptotically as

$$
\Phi_{n}^{\text {cum }}=\rho_{0}\left[\sigma \sqrt{\frac{2 n}{\pi}}-\epsilon+\frac{\mu_{4}+3 \sigma^{4}}{12 \sigma^{3}} \sqrt{\frac{1}{2 \pi n}}+\mathcal{O}\left(n^{-1}\right)\right],
$$

where $\mu_{4}=\int_{-\infty}^{\infty} f(x) x^{4} \mathrm{~d} x$ (assuming it is finite) and $\epsilon$ is the same quantity that appeared in the $3 \mathrm{D}$ flux problem in equation (9). The quantity $\epsilon$ however does not appear in the expression for the one-dimensional (1D) instantaneous flux as it does in three dimensions (at least up to $\left.\mathcal{O}\left(n^{-3 / 2}\right)\right)$.

The paper is organized as follows. In section 2, we set up the basic integral equation satisfied by the time-dependent density profile for an arbitrary jump distribution in three dimensions and show how one can reduce it to a one-dimensional integral equation. We then briefly describe the exact solution to this integral equation first obtained in [8]. In section 3 , we use this solution to calculate $\Phi_{n}^{\text {cum }}$ and from that derive the main result in equation (6). In the process, we also prove analytically some other conjectures made in [4] on the basis of numerics. Section 4 deals with the expressions for the flux in one dimension. Finally we conclude in section 5 with a summary and outlook. Some of the details of the calculations are relegated to the appendix.

\section{The integral equation for the density profile and its exact solution}

Let $\rho_{n}(\vec{r})$ denote the density of particles at position $\vec{r}$ outside the sphere of radius $R$ at time step $n$. Initially we have $\rho_{0}(\vec{r})=\rho_{0}$ for all $r>R$. The density stays zero inside the sphere since it is a trap. A particle at position $\vec{r}^{\prime}$ (with $r^{\prime}>R$ ) jumps in one time step to a new position $\vec{r}$ and the jump distance $\left(\vec{r}-\vec{r}^{\prime}\right)$ is drawn at each step independently from an isotropic distribution $W\left(\left|\vec{r}-\vec{r}^{\prime}\right|\right)$ that depends only on the magnitude of the jump length, but not on its direction. The function $W(u)$ is normalized in the three-dimensional space, i.e.,

$$
4 \pi \int_{0}^{\infty} W(u) u^{2} \mathrm{~d} u=1
$$

The time evolution of the density field $\rho_{n}(\vec{r})$ is clearly Markovian and is governed by the integral equation

$$
\rho_{n}(\vec{r})=\int_{r^{\prime} \geqslant R} \rho_{n-1}\left(\vec{r}^{\prime}\right) W\left(\left|\vec{r}-\vec{r}^{\prime}\right|\right) \mathrm{d} \vec{r}^{\prime}
$$

The initial condition is spherically symmetric and the evolution equation preserves this symmetry, hence $\rho_{n}(\vec{r})=\rho_{n}(r)$ at all steps $n$. We then have

$$
\rho_{n}(r)=\int_{r^{\prime} \geqslant R} \rho_{n-1}\left(r^{\prime}\right) W\left(\left|\vec{r}-\vec{r}^{\prime}\right|\right) \mathrm{d} \vec{r}^{\prime}
$$


Using $\left|\vec{r}-\vec{r}^{\prime}\right|=\sqrt{r^{2}+r^{\prime 2}-2 r r^{\prime} \cos \theta}$ where $\theta$ is the angle between $\vec{r}$ and $\vec{r}^{\prime}$ and $\mathrm{d} \vec{r}^{\prime}=$ $2 \pi r^{\prime 2} \sin \theta \mathrm{d} r^{\prime} \mathrm{d} \theta$ in three dimensions we get

$$
\begin{aligned}
\rho_{n}(r) & =2 \pi \int_{R}^{\infty} \mathrm{d} r^{\prime} \rho_{n-1}\left(r^{\prime}\right) r^{\prime 2} \int_{0}^{\pi} W\left(\sqrt{r^{2}+r^{\prime 2}-2 r r^{\prime} \cos \theta}\right) \sin \theta \mathrm{d} \theta \\
& =\frac{2 \pi}{r} \int_{R}^{\infty} \mathrm{d} r^{\prime} r^{\prime} \rho_{n-1}\left(r^{\prime}\right) \int_{\left|r-r^{\prime}\right|}^{r+r^{\prime}} W(u) u \mathrm{~d} u .
\end{aligned}
$$

Let us define a new variable $F_{n}(r)=r \rho_{n}(r) / \rho_{0}$. Then $F_{n}(r)$ for $r \geqslant R$ evolves via the one-dimensional integral equation

$$
F_{n}(r)=2 \pi \int_{R}^{\infty} \mathrm{d} r^{\prime} F_{n-1}\left(r^{\prime}\right) \int_{\left|r-r^{\prime}\right|}^{r+r^{\prime}} W(u) u \mathrm{~d} u \quad \text { with } F_{0}(r)=r .
$$

A further simplification can be achieved by introducing a shift, i.e., defining $z=r-R$ (distance from the surface of the sphere) and writing $F_{n}(r)=F_{n}(R+z)=H_{n}(z)$ where we have suppressed the $R$ dependence in $H_{n}(z)$ for convenience. Then $H_{n}(z)$ for $z \geqslant 0$ evolves via

$$
H_{n}(z)=2 \pi \int_{0}^{\infty} \mathrm{d} z^{\prime} H_{n-1}\left(z^{\prime}\right) \int_{\left|z-z^{\prime}\right|}^{z+z^{\prime}+2 R} W(u) u \mathrm{~d} u
$$

with $H_{0}(z)=R+z$.

Equation (19) is very general and is valid for arbitrary isotropic jump distribution $W(\mid \vec{r}-$ $\left.\vec{r}^{\prime} \mid\right)$. However, the reduced effective kernel in the one-dimensional integral equation (19)namely $f\left(z, z^{\prime}\right)=2 \pi \int_{\left|z-z^{\prime}\right|}^{z+z^{\prime}+2 R} W(u) u \mathrm{~d} u$-is non-stationary since it depends on both $r$ and $r^{\prime}$ and not just on its difference $\left|r-r^{\prime}\right|$. It is difficult to solve this integral equation with a nonstationary kernel. To simplify further, we will henceforth assume that the jump distribution $W\left(z=\left|\vec{r}-\vec{r}^{\prime}\right|\right)$ is bounded above, i.e., it has a finite support with an upper cut-off $z_{\max }$. This means $W(z)=0$ for $z>z_{\max }$. If we further assume that $z_{\max }<2 R$, it follows that one can replace the upper limit of integration in $f\left(z, z^{\prime}\right)$ by $\infty$ and equation (19) simplifies to one with a stationary kernel,

$$
H_{n}(z)=\int_{0}^{\infty} \mathrm{d} z^{\prime} H_{n-1}\left(z^{\prime}\right) f\left(z-z^{\prime}\right) \mathrm{d} z^{\prime} \quad \text { with } H_{0}(z)=R+z,
$$

where $f(x)$ is a symmetric non-negative function

$$
f(x)=2 \pi \int_{|x|}^{\infty} W(u) u \mathrm{~d} u .
$$

Moreover, it is easy to show from equation (21), via integration by parts and then using equation (14), that $f(x)$ is normalized to unity: $\int_{-\infty}^{\infty} f(x) \mathrm{d} x=1$. Thus $f(x)$ can be regarded as a probability density function. For later purposes, it would further be convenient to break the solution of equation (20) into two $R$-independent parts: $H_{n}(z)=Q_{n}(z)+R q_{n}(z)$ where $Q_{n}(z)$ and $q_{n}(z)$ evolve by the same integral equation, albeit with different initial conditions:

$$
\begin{aligned}
& Q_{n}(z)=\int_{0}^{\infty} \mathrm{d} z^{\prime} Q_{n-1}\left(z^{\prime}\right) f\left(z-z^{\prime}\right) \mathrm{d} z^{\prime} \quad \text { with } Q_{0}(z)=z, \\
& q_{n}(z)=\int_{0}^{\infty} \mathrm{d} z^{\prime} q_{n-1}\left(z^{\prime}\right) f\left(z-z^{\prime}\right) \mathrm{d} z^{\prime} \quad \text { with } q_{0}(z)=1 .
\end{aligned}
$$

Once the solutions to these two equations are known, the density profile $\rho_{n}(r)$ can be obtained via

$$
\rho_{n}(r)=\frac{\rho_{0}}{r}\left[Q_{n}(r-R)+R q_{n}(r-R)\right]
$$


The solutions to the two integral equations in equations (22) and (23) were obtained explicitly (in the Laplace domain) in [8]. We will not provide the derivation here, but just mention the main results. It was shown that the two following Laplace transforms

$$
\hat{\psi}_{\mathrm{I}}(u, s)=\int_{0}^{\infty}\left(\sum_{n=0}^{\infty} q_{n}(z) s^{n}\right) \mathrm{e}^{-u z} \mathrm{~d} z
$$

and

$$
\hat{\psi}_{\mathrm{II}}(u, s)=\int_{0}^{\infty}\left(\sum_{n=0}^{\infty} Q_{n}(z) s^{n}\right) \mathrm{e}^{-u z} \mathrm{~d} z,
$$

have the explicit solutions [8]

$$
\hat{\psi}_{\mathrm{I}}(u, s)=\frac{1}{u} \phi(0, s) \phi(u, s)
$$

and

$$
\hat{\psi}_{\mathrm{II}}(u, s)=\frac{1}{u}\left[\frac{1}{u} \phi(0, s)-\phi^{\prime}(0, s)\right] \phi(u, s),
$$

where the prime indicates differentiation with respect to $u, \phi(0, s)=(1-s)^{-1 / 2}$, and

$$
\begin{aligned}
\phi(u, s)=\frac{1}{\sqrt{1-s}+\sigma u \sqrt{s / 2}} & \\
& \times \exp \left[-\frac{u}{\pi} \int_{0}^{\infty} \frac{\mathrm{d} k}{u^{2}+k^{2}} \ln \left(\frac{1-s \hat{f}(k)}{1-s+s \sigma^{2} k^{2} / 2}\right)\right],
\end{aligned}
$$

where as before $\hat{f}(k)=\int_{-\infty}^{\infty} f(x) \mathrm{e}^{\mathrm{i} k x} \mathrm{~d} x$ is the Fourier transform of $f(x)$.

Using these solutions in equation (24) one can in principle calculate the full density profile at all times $n$. In [8], we have shown how to obtain the stationary solution (the $n \rightarrow \infty$ limit) explicitly. In the next section, we show how to obtain the two leading terms in the expression for flux using the explicit solutions mentioned above.

\section{Calculation of the discrete flux}

In this section our aim is to compute the expression for the discrete flux, in particular to prove the two leading terms in equation (6). To proceed, it turns out to be convenient to compute not the instantaneous flux $\Phi(t)$ as in equation (6) but rather the cumulative flux $\Phi^{\text {cum }}(t)$ up to time $t$. We also replace $t$ by the subscript $n$ to reflect the discrete nature of time in this problem. From (6) we expect

$$
\Phi_{n}^{\mathrm{cum}} \sim 4 \pi(R-\epsilon) D \rho_{0}\left[n+\frac{2(R-\epsilon) \sqrt{n}}{\sqrt{\pi D}}+\mathcal{O}(1)\right],
$$

where the correction term $\mathcal{O}(1)$ is effectively the constant of integration.

To prove the result in equation (30), we note that the cumulative flux up to time $n$ is simply the total number of particles absorbed by the sphere up to time $n$ and is given precisely by the 'missing' mass at that time:

$$
\begin{aligned}
\Phi_{n}^{\text {cum }} & =\int_{R}^{\infty} 4 \pi r^{2}\left[\rho_{0}-\rho_{n}(r)\right] \mathrm{d} r \\
& =4 \pi \rho_{0} \int_{R}^{\infty}\left[r^{2}-r F_{n}(r)\right] \mathrm{d} r \\
& =4 \pi \rho_{0} \int_{0}^{\infty}\left[(z+R)^{2}-(z+R)\left(Q_{n}(z)+R q_{n}(z)\right)\right] \mathrm{d} z
\end{aligned}
$$


where in line two we used $F_{n}(r)=r \rho_{n}(r) / \rho_{0}$ and in the third line we made the shift $z=r-R$ and used $F_{n}(z+R)=H_{n}(z)=\left(Q_{n}(z)+R q_{n}(z)\right)$ as explained in the previous section. Here $Q_{n}(z)$ and $q_{n}(z)$ are the solutions to equations (22) and (23) whose Laplace transforms are given explicitly in equations (28) and (27) respectively. So, our job is to use these explicit solutions in the expression in equation (31) and obtain the two leading terms for large $n$. The rest of the section is devoted precisely to this technical task.

Simplifying (31) further, we find

$$
\begin{aligned}
\Phi_{n}^{\text {cum }} & =4 \pi \rho_{0} \int_{0}^{\infty}(z+R)\left[z+R-\left(Q_{n}(z)+R q_{n}(z)\right)\right] \mathrm{d} z \\
& =4 \pi \rho_{0} \int_{0}^{\infty}(z+R)\left[\left(z-Q_{n}(z)\right)+R\left(1-q_{n}(z)\right)\right] \mathrm{d} z \\
& =4 \pi \rho_{0}\left[J_{n}^{(1)}+R\left(J_{n}^{(2)}+J_{n}^{(3)}\right)+R^{2} J_{n}^{(4)}\right],
\end{aligned}
$$

where

$$
\begin{aligned}
& J_{n}^{(1)}=\int_{0}^{\infty} z\left(z-Q_{n}(z)\right) \mathrm{d} z, \\
& J_{n}^{(2)}=\int_{0}^{\infty}\left(z-Q_{n}(z)\right) \mathrm{d} z, \\
& J_{n}^{(3)}=\int_{0}^{\infty} z\left(1-q_{n}(z)\right) \mathrm{d} z, \\
& J_{n}^{(4)}=\int_{0}^{\infty}\left(1-q_{n}(z)\right) \mathrm{d} z .
\end{aligned}
$$

One can verify that, for the case of the Rayleigh flight of jump length $\ell$ (11), the instantaneous flux $\Phi_{n}=\Phi_{n}^{\text {cum }}-\Phi_{n-1}^{\text {cum }}$ calculated from the above formulae agrees with the flux equation (13) of [4]:

$$
\Phi_{n}=4 \pi R D \rho_{0}\left[a^{(1)}(n)-(\ell / R) b^{(1)}(n)+(R / \ell) a^{(2)}(n)-b^{(2)}(n)\right]
$$

where

$a^{(1)}(n)=3 \int_{0}^{1}(1-z) Q_{n}(z) \mathrm{d} z, \quad b^{(1)}(n)=\frac{3}{2} \int_{0}^{1}(1-z)^{2} Q_{n}(z) \mathrm{d} z$,

$a^{(2)}(n)=3 \int_{0}^{1}(1-z) q_{n}(z) \mathrm{d} z, \quad b^{(2)}(n)=\frac{3}{2} \int_{0}^{1}(1-z)^{2} q_{n}(z) \mathrm{d} z$.

Now, to calculate the integrals $J_{n}^{(k)}$ for a general jump-length distribution, we proceed as follows. We first Taylor-expand $\phi(u, s)$ defined in equation (29) in powers of $u$ for small $u$. This gives

$$
\begin{aligned}
\phi(u, s)= & \frac{\phi(0, s)}{1+a(s) u} \mathrm{e}^{-u I_{2}(s)+u^{3} I_{4}(s)+\cdots} \\
& =\phi(0, s)\left[1-\left[a(s)+I_{2}(s)\right] u+\left[a(s)^{2}+a(s) I_{2}(s)+\frac{1}{2} I_{2}(s)^{2}\right] u^{2}+\cdots\right],
\end{aligned}
$$

where

$$
\begin{aligned}
& \phi(0, s)=(1-s)^{-1 / 2}, \\
& a(s)=\frac{\sigma \sqrt{s}}{\sqrt{2(1-s)}}, \\
& I_{n}(s)=\frac{1}{\pi} \int_{0}^{\infty} \frac{\mathrm{d} k}{k^{n}} \ln \left(\frac{1-s \hat{f}(k)}{1-s+s \sigma^{2} k^{2} / 2}\right), \quad n \leqslant 4 .
\end{aligned}
$$


Note that the analytic expansion in small $u$ in the first line of equation (39) is valid up to $\mathrm{O}\left(u^{3}\right)$ and the ... refers to non-analytic higher-order terms. For our purpose, we need terms only up to $\mathrm{O}\left(u^{3}\right)$.

Next, we carry out a Taylor-series expansion of $\hat{\psi}_{\mathrm{I}}$ and $\hat{\psi}_{\mathrm{II}}$ in powers of $u$ :

$$
\begin{gathered}
\hat{\psi}_{\mathrm{I}}(u, s)=\frac{1}{u(1-s)}-\frac{a(s)+I_{2}(s)}{1-s}+\frac{\left[a(s)^{2}+a(s) I_{2}(s)+\frac{1}{2} I_{2}(s)^{2}\right] u}{1-s}+\cdots \\
\hat{\psi}_{\mathrm{II}}(u, s)= \\
\quad \frac{1}{u^{2}(1-s)}-\frac{a(s) I_{2}(s)+\frac{1}{2} I_{2}(s)^{2}}{1-s} \\
\quad+\frac{\left[a(s)^{2} I_{2}(s)+a(s) I_{2}(s)^{2}+\frac{1}{3} I_{2}(s)^{3}+I_{4}(s)\right] u}{1-s}+\cdots
\end{gathered}
$$

and rewrite $\hat{\psi}_{\mathrm{I}}$ and $\hat{\psi}_{\mathrm{II}}$ in a way that separates off the singular part in $u$ :

$$
\begin{aligned}
& \hat{\psi}_{\mathrm{I}}(u, s)=\frac{1}{u(1-s)}-\sum_{n=0}^{\infty} s^{n} \int_{0}^{\infty}\left(1-q_{n}(z)\right) \mathrm{e}^{-u z} \mathrm{~d} z \\
& \hat{\psi}_{\mathrm{II}}(u, s)=\frac{1}{u^{2}(1-s)}-\sum_{n=0}^{\infty} s^{n} \int_{0}^{\infty}\left(z-Q_{n}(z)\right) \mathrm{e}^{-u z} \mathrm{~d} z,
\end{aligned}
$$

so that the integrals remain convergent when $u \rightarrow 0$. Expanding the exponentials $\mathrm{e}^{-u z}$ in powers of $u$, we get directly the generating functions of the desired integrals $J_{n}^{(k)}$. For example, $\sum s^{n} J_{n}^{(4)}$ follows from (minus) the coefficient of $u^{0}$ in $\hat{\psi}_{\mathrm{I}}(u, s)$. Thus, from equation (43) we deduce

$$
\sum_{n=0}^{\infty} s^{n} J_{n}^{(4)}=\frac{a(s)+I_{2}(s)}{1-s}=\frac{\sigma \sqrt{s}}{\sqrt{2}(1-s)^{3 / 2}}+\frac{I_{2}(s)}{1-s}
$$

We extract the large- $n$ behaviour of $J_{n}^{(k)}$ by looking at the behaviour of the generating function for $s \rightarrow 1$. To carry out expansions to higher order, we use (see the appendix)

$$
\frac{\sqrt{s}}{(1-s)^{3 / 2}}=\sum_{n=0}^{\infty} C_{n} s^{n}, \quad C_{n} \sim 2 \sqrt{\frac{n}{\pi}}+\frac{1}{4 \sqrt{\pi n}} \quad \text { as } n \rightarrow \infty .
$$

We also show in the appendix that

$$
I_{2}(s) \sim I_{2}(1)+\frac{\sqrt{2} \mu_{4}}{24 \sigma^{3}}(1-s)^{1 / 2}+\mathcal{O}(1-s),
$$

and

$$
I_{4}(s) \sim-\frac{\mu_{4}}{24 \sqrt{2} \sigma}(1-s)^{-1 / 2}+\mathcal{O}(1),
$$

as $s \rightarrow 1$, where $\mu_{4}=\int_{-\infty}^{\infty} x^{4} f(x) \mathrm{d} x$ is the fourth moment of the projected jump distribution $f(x)$.

Putting these results into (47), and using the Taylor-series expansions of $(1-s)^{1 / 2}$ and $(1-s)^{-1 / 2}$, we find

$$
J_{n}^{(4)} \sim \sigma \sqrt{\frac{2 n}{\pi}}-\epsilon+\frac{\mu_{4}+3 \sigma^{4}}{12 \sigma^{3}} \sqrt{\frac{1}{2 \pi n}}+\mathcal{O}\left(n^{-1}\right),
$$

where we have used $\epsilon=-I_{2}(1)$.

Likewise, $\sum s^{n} J_{n}^{(3)}$ follows from the coefficient of $u^{1}$ in $\Psi_{\mathrm{I}}(u, s)$ :

$$
\sum_{n=0}^{\infty} s^{n} J_{n}^{(3)}=\frac{a(s)^{2}+a(s) I_{2}(s)+\frac{1}{2} I_{2}(s)^{2}}{1-s}=\frac{\sigma^{2} s}{2(1-s)^{2}}+\frac{\sigma \sqrt{s} I_{2}(s)}{\sqrt{2}(1-s)^{3 / 2}}+\frac{\frac{1}{2} I_{2}(s)^{2}}{1-s}
$$


and using (49), we find

$$
J_{n}^{(3)} \sim \frac{\sigma^{2} n}{2}-\sigma \epsilon \sqrt{\frac{2 n}{\pi}}+\frac{1}{2} \epsilon^{2}+\frac{\mu_{4}}{24 \sigma^{2}}+\mathcal{O}\left(n^{-1 / 2}\right) .
$$

In the same way, $\sum s^{n} J_{n}^{(2)}$ follows from the coefficient of $u^{0}$ in $\Psi_{\mathrm{II}}(u, s)$ :

$$
\sum_{n=0}^{\infty} s^{n} J_{n}^{(2)}=\frac{a(s) I_{2}(s)+\frac{1}{2} I_{2}(s)^{2}}{1-s}=\frac{\sigma \sqrt{s} I_{2}(s)}{\sqrt{2}(1-s)^{3 / 2}}+\frac{\frac{1}{2} I_{2}(s)^{2}}{1-s},
$$

which implies

$$
J_{n}^{(2)} \sim-\sigma \epsilon \sqrt{\frac{2 n}{\pi}}+\frac{1}{2} \epsilon^{2}+\frac{\mu_{4}}{24 \sigma^{2}}+\mathcal{O}\left(n^{-1 / 2}\right) .
$$

Finally, $\sum s^{n} J_{n}^{(1)}$ follows from the coefficient of $u^{1}$ in $\Psi_{\mathrm{II}}(u, s)$ :

$$
\begin{aligned}
\sum_{n=0}^{\infty} s^{n} J_{n}^{(1)} & =\frac{a(s)^{2} I_{2}(s)+a(s) I_{2}(s)^{2}+\frac{1}{3} I_{2}(s)^{3}+I_{4}(s)}{1-s} \\
& =\frac{\sigma^{2} s I_{2}(s)}{2(1-s)^{2}}+\frac{\sigma \sqrt{s} I_{2}(s)^{2}}{\sqrt{2}(1-s)^{3 / 2}}+\frac{\frac{1}{3} I_{2}(s)^{3}}{1-s}+\frac{I_{4}(s)}{1-s} .
\end{aligned}
$$

Note that the first term on the right-hand side above behaves as

$$
\frac{\sigma^{2} s I_{2}(s)}{2(1-s)^{2}} \sim \frac{\sigma^{2} s I_{2}(1)}{2(1-s)^{2}}+\frac{s \sqrt{2} \mu_{4}}{48 \sigma(1-s)^{3 / 2}},
$$

and the last term of equation (56) behaves as

$$
\frac{I_{4}(s)}{1-s} \sim-\frac{s \sqrt{2} \mu_{4}}{48 \sigma(1-s)^{3 / 2}}
$$

as $s \rightarrow 1$, so between these two expressions, the terms containing $\mu_{4}$ exactly cancel out. With the remaining terms of (56), we find

$$
J_{n}^{(1)} \sim \frac{\sigma^{2} \epsilon n}{2}-\sigma \epsilon^{2} \sqrt{\frac{2 n}{\pi}}+\mathcal{O}(1)
$$

The $I_{2}(s)^{3}$ term in equation (56) does not contribute to this order.

Finally, putting the four results equations (51), (53), (55) and (59) into equation (32), we find, after some algebra,

$$
\begin{aligned}
\Phi_{n}^{\text {cum }} & =4 \pi \rho_{0}\left[J_{n}^{(1)}+R\left(J_{n}^{(2)}+J_{n}^{(3)}\right)+R^{2} J_{n}^{(4)}\right] \\
& =2 \pi \rho_{0}(R-\epsilon) \sigma^{2}\left[n+2(R-\epsilon) \sqrt{\frac{2 n}{\pi \sigma^{2}}}+\mathcal{O}(1)\right]
\end{aligned}
$$

which is precisely in the form of equation (30). Then $\Phi_{n}$ follows from $\Phi_{n}=\Phi_{n}^{\text {cum }}-\Phi_{n-1}^{\text {cum }}$, and the result is exactly in the form of equation (6) with $D=\sigma^{2} / 2$. This is the expected form of $D$ given that $\sigma$ is the standard deviation of the one-dimensional projection of the three-dimensional walk. (For example, for the Rayleigh flight, this corresponds to the familiar formula $D=\ell^{2} / 6$ [3].) This completes the proof of our main result, equation (6).

We close this section with the following observation. If we take the difference between (52) and (54), we find

$$
\sum_{n=0}^{\infty} s^{n}\left(J_{n}^{(3)}-J_{n}^{(2)}\right)=\frac{\sigma^{2} s}{2(1-s)^{2}}
$$


which implies

$$
J_{n}^{(3)}-J_{n}^{(2)}=\int_{0}^{\infty}\left(Q_{n}(z)-z q_{n}(z)\right) \mathrm{d} z=\frac{n \sigma^{2}}{2},
$$

exactly for all $n$. For the Rayleigh jump case, the right-hand side is $n \ell^{2} / 6$, and in that case this is the cumulative equivalent to the identity $a^{(1)}(n)=1-b^{(2)}(n)$ observed numerically in [4]. Thus, we have proven that result, and shown that it can be generalized for an arbitrary jump distribution, with the difference given in equation (62) depending only upon the standard deviation $\sigma$ of the jump distribution.

\section{One dimension}

Let us now consider a one-dimensional system where the origin represents a trap and initially we have particles distributed uniformly over the positive line $z>0$ with density $\rho_{0}$. Each particle jumps at every time step and the jump distance $u$ is drawn from a symmetric jump distribution $f(u)$. Whenever a particle crosses over the origin to the negative side it gets trapped by the origin. We ask similar questions as in $d=3$ : what is the density profile $\rho_{n}(z)$ at step $n$ and what is the instantaneous flux $\Phi_{n}$ at step $n$ ?

Due to the recurrent nature of the random walks in $d=1$, it is clear that eventually all the particles will be absorbed by the origin and hence $\rho_{\infty}(z)=0$ for all $z \geqslant 0$. This is a major difference from the $3 \mathrm{D}$ case. In one dimension, the density profile for $z>0$ evolves in time via the integral equation

$$
\rho_{n}(z)=\int_{0}^{\infty} \rho_{n-1}\left(z^{\prime}\right) f\left(z-z^{\prime}\right) \mathrm{d} z^{\prime} \quad \text { with } \rho_{0}(z)=\rho_{0} .
$$

Clearly, $\rho_{n}(z)=\rho_{0} q_{n}(z)$ where $q_{n}(z)$ satisfies the integral equation (23) with the initial condition $q_{0}(z)=1$ for all $z>0$, and we already know its solution from section 2 . Note that $q_{n}(z)$ can also be interpreted [10] as the probability that a random walker starting at $z>0$ and undergoing discrete jumps drawn from $f(z)$ does not cross the origin during the first $n$ steps, as discussed in [10].

Regarding the flux, the cumulative amount $\Phi_{n}^{\text {cum }}$ is given by the missing mass at step $n$

$$
\Phi_{n}^{\text {cum }}=\int_{0}^{\infty}\left[\rho_{0}-\rho_{n}(z)\right] \mathrm{d} z=\rho_{0} \int_{0}^{\infty}\left[1-q_{n}(z)\right] \mathrm{d} z .
$$

Now, the integral $\int_{0}^{\infty}\left[1-q_{n}(z)\right] \mathrm{d} z$ is precisely $J_{n}^{(4)}$ of equation (36) whose asymptotic behaviour is given in equation (51). The integral $\int_{0}^{\infty}\left[1-q_{n}(z)\right] \mathrm{d} z$ also represents the expected maximum $E\left[M_{n}\right]$ up to $n$ steps of a random walker starting at the origin and undergoing jumps drawn from $f(z)$ at every time step [10]. Thus, we have a rather nice relationship between the cumulative flux and the expected maximum,

$$
\Phi_{n}^{\text {cum }}=\rho_{0} E\left[M_{n}\right]=\rho_{0}\left[\sigma \sqrt{\frac{2 n}{\pi}}-\epsilon+\frac{\mu_{4}+3 \sigma^{4}}{12 \sigma^{3}} \sqrt{\frac{1}{2 \pi n}}+\mathcal{O}\left(n^{-1}\right)\right]
$$

where $\epsilon$ is the same quantity in equation (9) that appears in the expression for cumulative flux in three dimensions. However, while in three dimensions $\epsilon$ retains its appearance in the expression for the instantaneous flux $\Phi_{n}=\Phi_{n}^{\text {cum }}-\Phi_{n-1}^{\text {cum }}$, in one dimension it disappears from $\Phi_{n}$, at least up to this order. 
That is, from equation (65), we have

$$
\begin{aligned}
\Phi_{n}=\Phi_{n}^{\text {cum }}- & \Phi_{n-1}^{\text {cum }}=\rho_{0}\left(\sigma \sqrt{\frac{2}{\pi}}\left[n^{1 / 2}-(n-1)^{1 / 2}\right]+\frac{\mu_{4}+3 \sigma^{4}}{12 \sigma^{3} \sqrt{2 \pi}}\left[n^{-1 / 2}-(n-1)^{-1 / 2}\right]\right) \\
= & \rho_{0} \sigma \sqrt{\frac{2}{\pi}}\left(n^{1 / 2}\left[1-\left(1-\frac{1}{2 n}-\frac{1}{8 n^{2}}+\cdots\right)\right]\right. \\
& \left.+\frac{\mu_{4}+3 \sigma^{4}}{24 \sigma^{4}} n^{-1 / 2}\left[1-\left(1+\frac{1}{2 n}+\cdots\right)\right]\right) \\
= & \rho_{0} \frac{\sigma}{\sqrt{2 \pi}}\left(n^{-1 / 2}+\frac{3 \sigma^{4}-\mu_{4}}{24 \sigma^{4}} n^{-3 / 2}+\cdots\right)
\end{aligned}
$$

which does not involve $\epsilon$. Note that the coefficient of the last term is proportional to the 'excess', defined as $\gamma_{2}=\mu_{4} / \sigma^{4}-3$. This quantity equals zero for a Gaussian distribution.

For the case of the uniform $f(x)$ of equation (11), we have $\sigma^{2}=\ell^{2} / 3$ and $\mu_{4}=\ell^{4} / 5$, and from equation (66), it follows that the flux is given by

$$
\Phi_{n}=\rho_{0} \ell \sqrt{\frac{1}{6 \pi}}\left(n^{-1 / 2}+\frac{1}{20} n^{-3 / 2}+\cdots\right) .
$$

This result proves one more formula that was conjectured in [4]. For the uniform $f(x), \Phi_{n}$ equals the $\rho_{0} \ell a^{(2)}(n) / 6$ where $a^{(2)}(n) / 6$ is given in equation (38), and in equation (21c) of [4] it was conjectured from the numerical results that

$$
a^{(2)}(n) / 6=(6 \pi)^{-1 / 2} n^{-1 / 2}+(1 / 20)(6 \pi)^{-1 / 2} n^{-3 / 2}+\mathcal{O}\left(n^{-5 / 2}\right) .
$$

Indeed, the above formula is identical to equation (67). Thus, we have proven equation (68), and in particular verified that the coefficient in the second term is exactly $1 / 20$.

Note, finally, for the uniform $f(x)$, equation (65) becomes

$$
\Phi_{n}^{\text {cum }}=\rho_{0} \ell\left[\sqrt{\frac{2}{3 \pi}} n^{1 / 2}-c+\frac{1}{5} \sqrt{\frac{2}{3 \pi}} n^{-1 / 2}+\mathcal{O}\left(n^{-1}\right)\right]
$$

where $c=0.29795219 \ldots$ as given in equation (7). This result agrees with the expectation of the maximum of a one-dimensional walker with uniform jump density $[9,10], E\left[M_{n}\right]$, which is identical to $\Phi_{n}^{\text {cum }}$ (up to the constant factor $\rho_{0}$ ) as mentioned above.

\section{Conclusions}

Thus, we have proven that equation (6) or its integrated form equation (30) applies to an arbitrary jump distribution $f(x)$. Here $f(x)$ is related to the radial projection of the threedimensional jump distribution $W\left(\left|\vec{r}-\vec{r}^{\prime}\right|\right)$ by equation (10) as long as $W(u)$ has a cutoff for large $u$. The generality of the flux in equation (6) implies that there is a universal long-time behaviour of the intermediate particle density given by

$$
\rho(r, t) \sim \rho_{0}\left(\frac{r-R+\epsilon}{r}\right)\left(1+\frac{R-\epsilon}{\sqrt{\pi D t}}\right),
$$

valid for large $t$ and $R \ll r \ll \sqrt{\pi D t}$. Only the length $\epsilon$ and diffusion coefficient $D$ depend upon the details of the jump distribution. Equation (70) is equivalent to equation (1) with $R$ replaced by $R-\epsilon$ and the complementary error function expanded to two terms. 
The integrated form of the flux of equation (30) brings in additional constants that do not appear in the instantaneous form, equation (6), including the quantity $\epsilon$ that appears in the onedimensional flux, equation (69). Indeed, $\epsilon$ defined in equation (9) is ubiquitous as it appears in many terms in the expansions of the integrals $J_{n}^{(k)}$ that apply to both one- and three-dimensional problems.

The integrated form of the flux also enters directly in the formula for the trap survival probability (the probability that an adsorbing sphere is not hit by a diffusing particle up to time $t): S(t)=\exp \left[-\Phi^{\mathrm{cum}}(t)\right]$. This quantity has been studied extensively and has applications to quenching of fluorescence reactions (e.g., [12-14]).

When the jump probability corresponds to the Rayleigh flight, $f(x)$ becomes the uniform jump distribution of equation (11), in which case $\epsilon$ becomes $0.29795219 \ldots \ell$ found previously $[4,8]$. For this model we have also provided proofs of the conjectured behaviour of the quantities $a^{(1)}(n), b^{(1)}(n), a^{(2)}(n)$, and $b^{(2)}(n)$ in equations (21a)-(21d) of [4] and proven the conjectured identity $a^{(1)}(n)=1-b^{(2)}(n)$. We did not derive expressions for the higherorder corrections of equations (21b) and (21d) of [4] that involve the empirically determined constant $d=0.0270103$, but these terms enter only in higher-order corrections to the flux.

We also proved the identity (62) for a general jump distribution. This identity relates the solution $q_{n}(z)$ with a linear initial condition to the solution $Q_{n}(z)$ with a constant initial condition, and implies a subtle relation between the corresponding one- and three-dimensional diffusion processes.

In this paper we have restricted ourselves to the spatial dimensions 1 and 3. A natural question is: what are the expressions for the density profile $\rho_{n}(r)$ and the cumulative flux $\Phi_{n}^{\text {cum }}$ in dimensions $d \neq 1,3$ ? Is it possible to compute the Milne extrapolation length $\epsilon$ for $d>3$ ? The reason we restricted ourselves only to $d=1$ and $d=3$ can be traced back to the basic integral equation (16). For $d=1$ and $d=3$, this integral equation can be reduced to a onedimensional integral equation (20) with a stationary kernel $f\left(z-z^{\prime}\right)$ that is symmetric. For $d \neq 1,3$ this step cannot be done and finding an exact solution of the integral equation seems difficult. For example, in $d=2$, using $\mathrm{d} \vec{r}^{\prime}=r^{\prime} \mathrm{d} r^{\prime} \mathrm{d} \theta$ in equation (16) one gets

$$
\rho_{n}(r)=\int_{R}^{\infty} \mathrm{d} r^{\prime} \rho_{n-1}\left(r^{\prime}\right) r^{\prime} \int_{0}^{2 \pi} W\left(\sqrt{r^{2}+r^{\prime 2}-2 r r^{\prime} \cos \theta}\right) \mathrm{d} \theta .
$$

If one performs the angular integration after the substitution $z^{2}=r^{2}+r^{\prime 2}-2 r r^{\prime} \cos \theta$ one gets the following integral equation:

$$
\rho_{n}(r)=\int_{R}^{\infty} \mathrm{d} r^{\prime} \rho_{n-1}\left(r^{\prime}\right) G\left(r, r^{\prime}\right)
$$

where the kernel $G\left(r, r^{\prime}\right)$ is a rather complicated function of both $r$ and $r^{\prime}$ :

$$
G\left(r, r^{\prime}\right)=2 r^{\prime} \int_{\left|r-r^{\prime}\right|}^{r+r^{\prime}} \frac{\mathrm{d} z z W(z)}{\sqrt{4 r^{2} r^{\prime 2}-\left(z^{2}-r^{2}-r^{\prime 2}\right)^{2}}} .
$$

Solving the integral equation (72) or the equivalent integral equations for $d>3$ remains a challenging problem.

\section{Acknowledgment}

RMZ acknowledges support from NSF grant DMS-0553487. 


\section{Appendix. Proof of equations (48) and (49)}

Proof of equation (48). For the terms that involve $a(s) /(1-s)$, we have for $s$ near the singular point at $s=1$,

$$
\begin{aligned}
\frac{\sqrt{s}}{(1-s)^{3 / 2}} & =\frac{(1-(1-s))^{1 / 2}}{(1-s)^{3 / 2}} \sim(1-s)^{-3 / 2}-\frac{1}{2}(1-s)^{-1 / 2} \\
& =\sum_{n=0}^{\infty}(2 n+1)\left(\begin{array}{c}
2 n \\
n
\end{array}\right) \frac{s^{n}}{2^{2 n}}-\frac{1}{2} \sum_{n=0}^{\infty}\left(\begin{array}{c}
2 n \\
n
\end{array}\right) \frac{s^{n}}{2^{2 n}} \\
& =\sum_{n=0}^{\infty} C_{n} s^{n} .
\end{aligned}
$$

Now, for large $n$,

$$
\frac{1}{2^{2 n}}\left(\begin{array}{c}
2 n \\
n
\end{array}\right) \sim \sqrt{\frac{1}{\pi n}}\left[1-\frac{1}{8 n}+\cdots\right] .
$$

Therefore, we have

$$
C_{n} \sim \frac{1}{\sqrt{\pi n}}\left[1-\frac{1}{8 n}+\cdots\right]\left(2 n+1-\frac{1}{2}\right)=2 \sqrt{\frac{n}{\pi}}+\frac{1}{4 \sqrt{\pi n}}+\cdots,
$$

thus contributing to the $n^{1 / 2}$ and $n^{-1 / 2}$ terms in the large- $n$ behaviour.

Derivation of equation (49). First differentiate equation (42) (for $n=2$ ) with respect to $s$, then put $s=1-\varepsilon$ where $\varepsilon$ is small. Next, inside the corresponding integrand make the change of variable $k=\sqrt{\varepsilon} y$ and then expand everything for small $\varepsilon$. Use $\hat{f}(k) \sim$ $1-\sigma^{2} k^{2} / 2+\mu_{4} k^{4} / 24$. What you get as $\varepsilon \rightarrow 0$ is the following:

$$
\frac{\mathrm{d} I_{2}}{\mathrm{~d} \varepsilon} \sim \frac{\mu_{4} \sqrt{2}}{12 \pi \sigma^{3}} \varepsilon^{-1 / 2} \int_{0}^{\infty} \frac{z^{2} \mathrm{~d} z}{\left(1+z^{2}\right)^{2}}
$$

The integral gives $\pi / 4$. Then integrating the above with respect to $\varepsilon$ one gets

$$
I_{2}(\varepsilon) \sim I_{2}(\varepsilon=0)+C_{1} \sqrt{\varepsilon}
$$

where $C_{1}=\sqrt{2} \mu_{4} /\left(24 \sigma^{3}\right)$.

For $I_{4}$, the integral is already singular as $\varepsilon \rightarrow 0$ and there is no need to differentiate. A direct analysis of the integral gives equation (50).

\section{References}

[1] Redner S 2001 A Guide to First-Passage Processes (Cambridge: Cambridge University Press) see also the list of errata at http://physics.bu.edu/ redner/

[2] Smoluchowski M 1916 Drei Vortrage uber Diffusion, Brownsche Bewegung und Koagulation von Kolloidteilchen Phys. Z. 17 557-71, 585-99

[3] Chandrasekhar S 1943 Stochastic problems in physics and astronomy Rev. Mod. Phys. 15 1-89

[4] Ziff R M 1991 Flux to a trap J. Stat. Phys. 65 1217-33

[5] Burschka M A and Titulaer U M 1981 The kinetic boundary layer theory for the Fokker-Planck equation with absorbing boundary J. Stat. Phys. 25 569-82

[6] Case K M and Zwiefel P W 1967 Linear Transport Theory (Reading, MA: Addison-Wesley)

[7] Williams M M R 1966 The Slowing Down and Thermalization of Neutrons (Amsterdam: North-Holland)

[8] Majumdar S N, Comtet A and Ziff R M 2006 Unified solution of the expected maximum of a discrete-time random walk and the discrete flux to a spherical trap J. Stat. Phys. 122 833-56

[9] Coffman E G, Flajolet P, Flato L and Hofri M 1998 The maximum of random walk and its application to rectangle packing Probab. Eng. Inform. Sci. 12 373-86 
[10] Comtet A and Majumdar S N 2005 Precise asymptotics for a random walker's maximum J. Stat. Mech. P06013

[11] Collins F C and Kimball G E 1949 Diffusion-controlled reaction rates J. Colloid Sci. 4 425-37

[12] Zhou H-X and Szabo A 1991 Comparison between molecular dynamics simulations and the Smoluchowski theory of reactions in a hard sphere liquid J. Chem. Phys. 95 5948-52

[13] Dong W and Andre J C 1994 Diffusion-controlled reactions. II. An approach based on a generalized diffusion equation J. Chem. Phys. 101 299-306

[14] Ibuki K and Ueno M 1997 Improved treatment of inertia and non-Markovian effects on short-time dynamics of diffusion-controlled reaction based on generalized diffusion equation J. Chem. Phys. 106 10113-22 